Band 1: Johannes Rakers, Kommunale Verschuldung 42,-

Band 3: Thomas Hahn, Standort-Entscheidung unter Unsicherheit

Band 4: Johann-Peter Thielenhaus, Strategische Personalentwicklungsplanung

Band 5: Friedrich Lutz, Das Beratungsteam

Band 6: Alexander Dehmel, Die besondere Stellung des US-Dollars

Band 7: Otmar Seibert, Regionale Einkommensparitäten in der Bundesrepublik Deutschland

Band 8: Adolf Rosenstock, Der Realzins

Band 9: Heinz-Jürgen Crössmann, Entscheidungsverhalten auf unvollkommenen Märkten

Band 10: Richard Bopp, Europäische Aufsicht über Kreditinstitute

Band 11: Herbert Runow, Zur Theorie und Messung der Verbraucherzufriedenheit

Band 12: Horst-Heinrich Koch, Die Verrechtlichung der regionalen Wirtschaftspolitik und ihre ökonomische Basis

Band 13: Hans-Peter Steinmetz, Die Koordinationsfunktion der Gemeinschaftsaufgabe „Verbesserung der regionalen Wirtschaftsstruktur" - Anspruch und Wirklichkeit -

Band 14: Michael Hopf, Informationen für Märkte und

Märkte für Informationen 
Leichsenring - Führungsinformationssysteme in Banken 
OIKOS - Studien zur Ökonomie - Band 25

Herausgegeben von Dr. Stephan M. Hess 
Hansjörg Leichsenring

\section{Führungsinformationssysteme in Banken}

Notwendigkeit, Konzeption und strategische Bedeutung 
CIP-Titelaufnahme der Deutschen Bibliothek

Leichsenring, Hansjörg:

Führungsinformationssysteme in Banken: Notwendigkeit, Konzeption und strategische Bedeutung/Hansjörg

Leichsenring. - Wiesbaden: Gabler, 1990

(Oikos; Bd. 25)

NE: GT

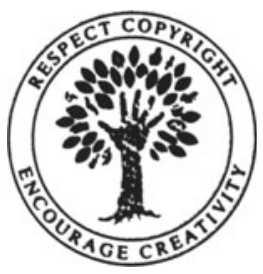

ISBN 978-3-663-00139-3

ISBN 978-3-663-00138-6 (eBook)

DOI 10.1007/978-3-663-00138-6

Der Gabler Verlag ist ein Unternehmen der Verlagsgruppe Bertelsmann International.

(C) Betriebswirtschaftlicher Verlag Dr. Th. Gabler GmbH, Wiesbaden 1990 Lektorat: Karlheinz Müssig

Das Werk einschlieBlich aller seiner Teile ist urheberrechtlich geschützt. Jede Verwertung auBerhalb der engen Grenzen des Urheberrechtsgesetzes ist ohne Zustimmung des Verlags unzulässig und strafbar. Das gilt insbesondere für Vervielfältigungen, Übersetzungen, Mikroverfilmungen und die Einspeicherung und Verarbeitung in elektronischen Systemen.

Satz: Satzstudio RESchulz, Dreieich-Buchschlag 
Meinen lieben Eltern 
There are always trends and tides that make new fortunes for the informated few...

(Aus einem Werbeprospekt für Informationsdienste) 


\section{Vorwort}

Die Frage, welche Informationen das Management einer Bank zur Erfüllung seiner Aufgaben benötigt, ist heute aktueller denn je. Ein Hauptproblem liegt dabei für viele Bankmanager nicht darin, daB sie über zuwenig Informationen verfügen, vielmehr fehlt ihnen der Zugang zu den ${ }_{n}$ richtigen “ Informationen. Im Zeichen des globalen Wandels der Finanzmärkte, wachsender Risiken bei vielen Bankgeschäften und neuer Konkurrenten auf dem Gebiet der Finanzdienstleistungen liegt eine entscheidende Aufgabe der Führung einer Bank darin, sich in angemessener Zeit den Informationsstand zu verschaffen, der sie dazu befähigt, nicht nur Probleme richtig zu entscheiden, sondern vor allem über die richtigen Probleme zu entscheiden.

Die vorliegende Arbeit will einen Beitrag leisten, die Informationsvielfalt im Bankbetrieb handhabbarer zu gestalten, indem eine Konzeption bankbetrieblicher Führungsinformationssysteme entwickelt und diskutiert wird, die geeignet erscheint, ihrer besonderen $\mathrm{Be}$ deutung für eine zukunftsgerichtete Bankführung gerecht zu werden.

An dieser Stelle möchte ich denjenigen danken, ohne deren Unterstützung die Arbeit nicht in der vorliegenden Form hätte realisiert werden können.

Mein bester Dank gilt dabei Herrn Professor Dr. Leo Schuster, der mir innerhalb des Instituts für Bankwirtschaft die Möglichkeit gab, an den unterschiedlichsten Projekten mitzuarbeiten, dabei praktische und wissenschaftliche Erfahrungen sammeln zu können und der mich in vielfältiger Art und Weise ermuntert hat, die vorliegende Schrift zu verfassen. Durch seine positive menschliche Haltung ist er für mich stets mehr als nur ein akademischer Lehrer und Vorgesetzter gewesen.

Herrn Professor Dr. Hans Siegwart danke ich für die bereitwillige Übernahme des Korreferates und seine freundliche Förderung meiner Arbeit. Die unter seiner Leitung durchgeführten Seminare waren stets eine sprudelnde Quelle für neue produktive Gedanken.

Meinen Kollegen am Institut für Bankwirtschaft der Hochschule St. Gallen danke ich für die vielen gemeinsam verbrachten Stunden, in denen ein gegenseitig befruchtender Gedankenaustausch weit über fachliche Themen hinaus zustande kam.

Danken möchte ich auch denen, deren Freundschaft mir Selbstvertrauen und Kraft gab, um über schwierige und unproduktive Phasen hinwegzukommen. 
Mein besonderer Dank aber gilt meinen Eltern, die mich in herzlicher Liebe und Zuneigung beim Finden des richtigen Lebensweges unterstützt haben. Ihnen sei diese Schrift in ebenso herzlicher Liebe gewidmet.

Hansjörg Leichsenring 


\section{Vorwort}

Seit Franz Kafkas Roman „Das SchloB“, spätestens aber seit Parkinson wissen wir, daB Bürokratien eigenstăndige Informations- und Desinformationssysteme entwickeln. Die Flut der Titel und Positionsbezeichnungen, wie sie in der Donau-Monarchie fast zur Persiflage perfekt betrieben wurde, war nicht feiner abgestimmt als die Titel-Hierarchie in vielen (meist amerikanischen) GroBkonzernen heute, wo sie Organisationsformen und Führungsstrukturen reflektieren sollen. Matrix-Organisationen, ntop-down “ und „bottom-up - Informationsflüsse, eigene Abteilungen für hausinterne Information und Kommunikation sowie groBe Entwicklungsstäbe für Management-Informations-Systeme zeigen, wie sehr göBere Unternehmen die Notwendigkeit verspüren, Führungsinformationssysteme durch eigene Abteilungen und Informationskanäle etablieren zu müssen.

Bedenkt man, daB das Bankgeschäft im wesentlichen ein Geschäft mit Informationen ist, und zwar mit Informationen über Geld und dessen Märkte, dann wird klar, daB der Bedarf an Führungsinformationen besonders groB ist. Dennoch hinkt der Informationsstand im Bereich der "weichen“ Informationen (qualitative Informationen) dem der "harten" Informationen (quantitative Informationen) nach. Hier geht es darum, dem alten Modell nachzueifern, wo die Bankangestellten noch in einem Zimmer zusammensaBen und jedes Gespräch allen zugänglich war. Der Versuch, dieses alte Vorbild in die Praxis des Unternehmens "Grobbank" zu übertragen, ist letzten Endes die Aufgabe, Führungsinformationssysteme zu schaffen.

Wie wichtig die Schaffung solcher Systeme ist, zeigt die Notwendigkeit, aktuellen Zugang zu allen Ebenen an Informationen zu haben, um die mit den Finanzinstrumenten einhergehenden Risiken unternehmerisch bewältigen und die sich ergebenden Chancen nutzen zu können. Leichsenring geht die Probleme analytisch an und zeigt, wie sich in der Praxis Modelle für Führungsinformationssysteme darstellen und umsetzen lassen. 


\title{
Inhaltsübersicht
}

\author{
Einleitung, Zielsetzung und Aufbau der Arbeit \\ I. Banken im Zeitalter der Informatization \\ II. Zielsetzung und Vorgehensweise
}

Teil Eins: Grundlagen von Führung und Information in Banken

I. Banken als Gegenstand der Untersuchung

II. Elemente der bankbetrieblichen Führung

III. Zusammenhang zwischen Führung und Information

Teil Zwei: Bedeutung des Faktors Information für die Banken

I. Informationsrelevante Eigenheiten der Bankleistung

II. Strukturwandel an den Finanzmärkten

III. Informationsmanagement als neue Herausforderung

Teil Drei: Entwicklung einer Konzeption bankbetrieblicher Führungsinformationssysteme

I. Grundkonzeption bankbetrieblicher Führungsinformationssysteme

II. Formale Konzeption bankbetrieblicher Führungsinformationssysteme

III. Inhaltliche Konzeption bankbetrieblicher Führungsinformationssysteme

IV. Bausteine bankbetrieblicher Führungsinformationssysteme

V. Hinweise zur Realisierung bankbetrieblicher Führungsinformationssysteme

Teil Vier: Strategische Relevanz bankbetrieblicher Führungsinformationssysteme

I. Führungsinformationssysteme aus Sicht der bankbetrieblichen Praxis

II. Aufgaben und Ziele der strategischen Führung in Banken

III. Wettbewerbsvorteile durch Einsatz bankbetrieblicher Führungsinformationssysteme

Zusammenfassung und SchluBbetrachtung

Literaturverzeichnis 


\section{Inhaltsverzeichnis}

\section{Einleitung, Zielsetzung und Aufbau der Arbeit}

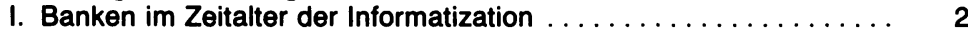

II. Zielsetzung und Vorgehensweise .................. 5

\section{Teil Eins: Grundlagen von Führung und}

$$
\text { Information in Banken }
$$

Vorbemerkungen zum ersten Teil . . . . . . . . . . . . . . 11

I. Banken als Gegenstand der Untersuchung . . . . . . . . . . . . . 11
A. Forschungsobjekt "Bank“

B. Systemansatz als integrativer Ansatz zur Unternehmensführung im Bankbetrieb

II. Elemente der bankbetrieblichen Führung $\ldots \ldots \ldots \ldots \ldots \ldots \ldots \ldots, 14$

A. Begriff der Führung $\ldots \ldots \ldots \ldots \ldots \ldots \ldots \ldots \ldots \ldots \ldots \ldots \ldots$

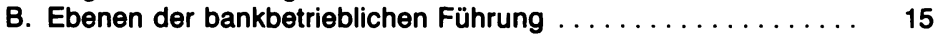

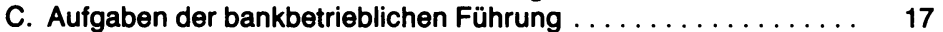

D. Funktionen der bankbetrieblichen Führung $\ldots \ldots \ldots \ldots \ldots \ldots 20$

E. Institutionen der bankbetrieblichen Führung $\ldots \ldots \ldots \ldots \ldots \ldots 22$

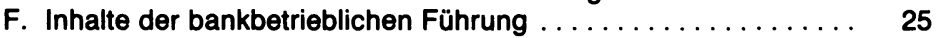

1. Geschäftspolitisches Zielsystem .............. 26

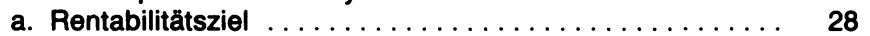

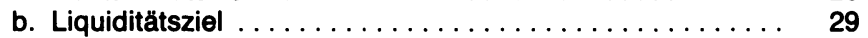

c. Das Sicherheitsziel ................... 31

d. Weitere bankpolitische Ziele .............. 32

2. Strategische Geschäftsfeldkonzeption ............ 33

a. Grundlagen der Konzeption strategischer Geschäftsfelder 33

b. Möglichkeiten der Bildung strategischer Geschäftsfelder in

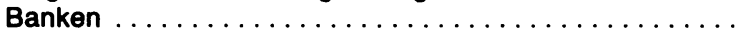

3. Geschäftspolitische Entscheidungen ............. 37

III. Zusammenhang zwischen Führung und Information ......... 39

A. Aspekte der bankbetrieblichen Information und Kommunikation . $\quad 39$

1. Information, betriebliche Information, Bericht und Führungsinformation .......................... 39

2. Kommunikation und betriebliche Kommunikation ...... 42

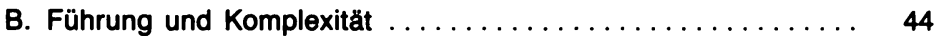

C. Führung als informationsverarbeitender ProzeB ......... 45

D. Grundprobleme der Information und Kommunikation in Banken . 49

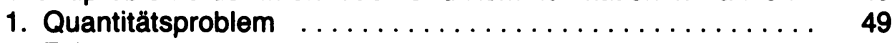

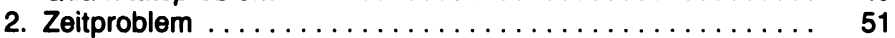

3. Qualitätsproblem ........................... 52

4. Kommunikationsproblem .................... 54

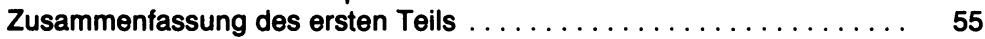


Teil Zwei: Bedeutung des Faktors Information für die Banken . . . . . Vorbemerkungen zum zweiten Teil . ................. 60

I. Informationsrelevante Eigenheiten der Bankleistung . . . . . . 61

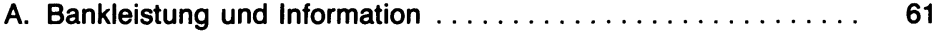

B. Stofflichkeit und Unstofflichkeit der Bankleistung . . . . . . . 63

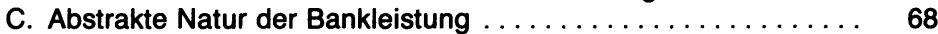

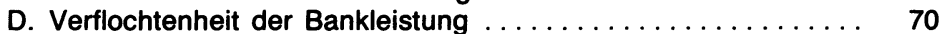

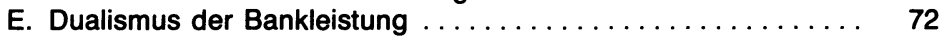

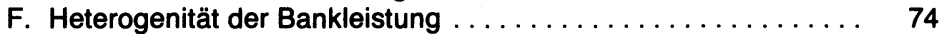

II. Strukturwandel an den Finanzmärkten und sein EinfluB auf die bankbetriebliche Informationsproblematik .............. 75

A. Strukturwandel am Markt für Finanzdienstleistungen . . . . . . 75

1. Informatization als übergeordneter Trend ........... 77

2. Wandel in der ökonomischen Umweltsphäre . . . . . . . . . 78

3. Wandel in der politisch-gesetzlichen Umweltsphäre . . . . . 83

4. Wandel in der technologischen Umweltsphäre ......... 84

5. Wandel in der sozio-kulturellen Umweltsphäre . . . . . . 87

B. Auswirkungen des Wandels auf die bankbetriebliche Führung .. 88

C. Auswirkungen des Wandels auf die bankbetriebliche Informationsproblematik

III. Informationsmanagement als neue Herausforderung . . . . . . . . . 93

A. Banken: ${ }_{n}$ Informationsverarbeiter par excellence $\ldots \ldots \ldots . .93$

B. Informationsmanagement als Herausforderung an die Banken .. 98

1. Elemente eines bankbetrieblichen Informationsmanagements 98

2. Management der Ressource "Information" in Banken ...... 100

3. Probleme des Technologieeinsatzes .............. 104

4. Herausforderung für das Bankmanagement . . . . . . . . 107

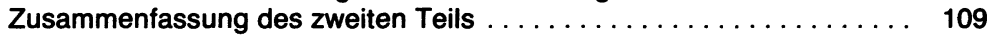

Teil Drei: Entwicklung einer Konzeption bankbetrieblicher

Führungsinformationssysteme

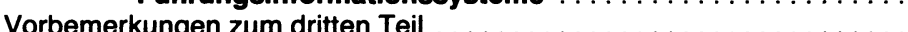

.

I. Grundkonzeption bankbetrieblicher Führungsinformationssysteme . 115

A. Forschungsaspekte im Bereich von Führungsinformationssyste-

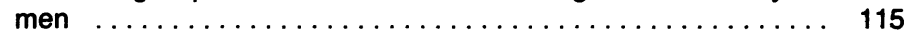

B. Begriff des Managementinformationssystems ......... 117

C. Managementinformationssystem versus Entscheidungsunterstüt-

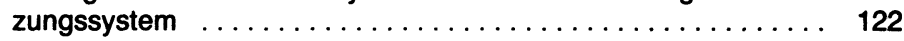

D. Betriebliches Informationssystem und Führungsinformationssystem

II. Formale Konzeption bankbetrieblicher

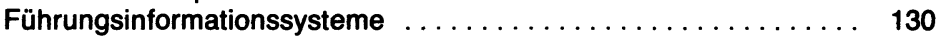

A. Grundsatz der Benutzeradäquanz . . . . . . . . . . 130

1. Relevanz .......................... 131

2. Stufengerechtigkeit $\ldots \ldots \ldots \ldots \ldots \ldots \ldots \ldots \ldots \ldots \ldots \ldots \ldots$

3. Flexibilität $\ldots \ldots \ldots \ldots \ldots \ldots \ldots \ldots \ldots \ldots \ldots \ldots \ldots \ldots \ldots \ldots \ldots \ldots$ 


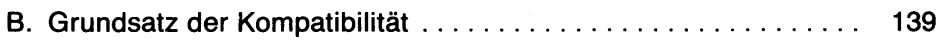

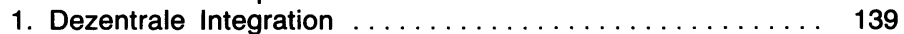

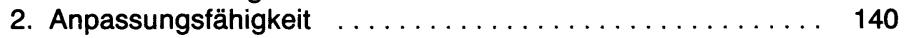

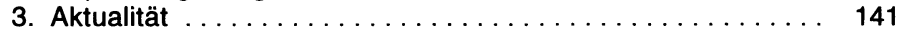

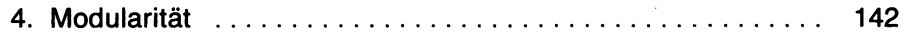

C. Grundsatz der konzeptionellen Vollständigkeit . . . . . . . 143

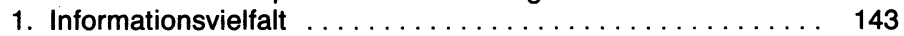

2. Multidimensionalität $\ldots \ldots \ldots \ldots \ldots \ldots \ldots \ldots \ldots \ldots \ldots$

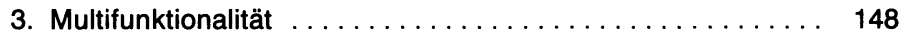

D. Grundsatz der Konsistenz . . . . . . . . . . . . . . . 149

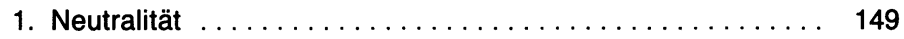

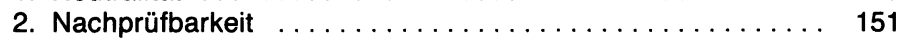

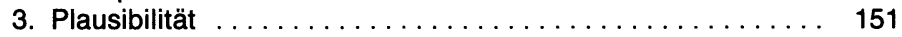

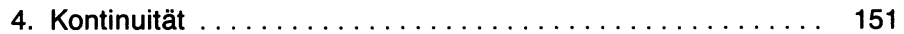

E. Grundsatz der Sicherheit $\ldots \ldots \ldots \ldots \ldots \ldots \ldots \ldots \ldots \ldots \ldots \ldots . \ldots \ldots$

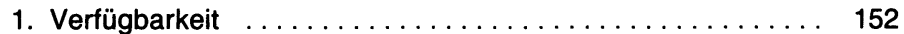

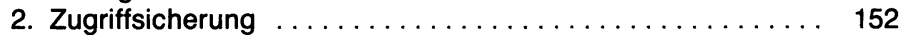

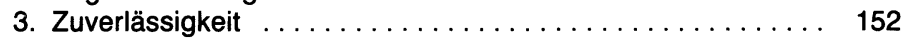

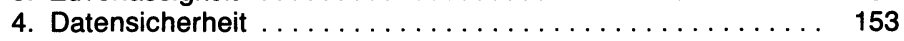

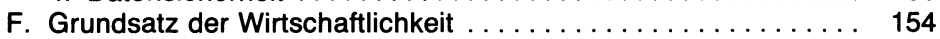

III. Inhaltliche Konzeption bankbetrieblicher Führungsinformationssysteme ................................... 155

A. Informationsbedarfsanalyse als zentraler Entwicklungsbestandteil 155

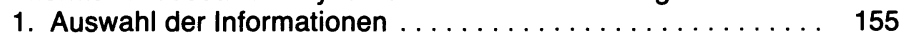

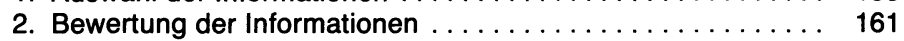

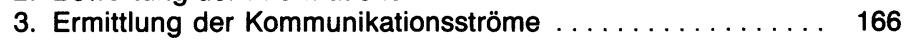

B. Informationsbedürfnisse des Bankmanagements . . . . . . . 169

C. Konsequenzen aus dem geschäftspolitischen Zielsystem . . . . . 173

1. Spezielle aus dem Ertragsziel resultierende Anforderungen . . 174

2. Spezielle aus dem Liquiditätsziel resultierende Anforderungen 175

3. Spezielle aus dem Sicherheitsziel resultierende Anforderungen 176

D. Konsequenzen aus der strategischen Geschäftsfeldkonzeption . 178

IV. Bausteine bankbetrieblicher Führungsinformationssysteme . . . . . 182

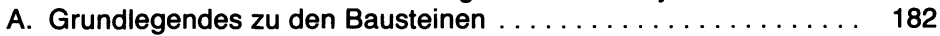

B. Bankrechnungswesen als zentraler Baustein . . . . . . . . 184

1. Problematik einer exakten Quantifizierung der bankbetrieblichen Gestehungskosten .................... 184

2. Grundaufbau der Bankkostenrechnung ........... 186

3. Betriebsstatistik als wichtiges Bindeglied .......... 188

4. Kalkulation der bankbetrieblichen Wertleistung ....... 190

a. Traditionelle Teilzinsspannenverrechnungsmethoden .... 191

b. Opportunitätszinskonzept als neuer Weg . .......... 193

5. Kalkulation der bankbetrieblichen Stück- oder Betriebsleistung 198

a. Problematik der Kalkulation der bankbetrieblichen Stückoder Betriebsleistung . . . . . . . . . . . . . . 198

b. Traditionelle Verfahren der Stückleistungskalkulation ... . 202

c. Moderne Verfahren der Stückleistungskalkulation ...... 203 
C. Berichtswesen als zentraler Baustein des bankbetrieblichen

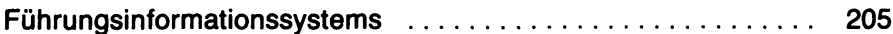

1. Verknüpfung durch das Planungs- und Analysesystem . . . . 205

a. Grundsätzliches zum Planungs- und Analysesystem . . . . 205

b. Durch das Planungs- und Analysesystem bereitgestellte Da-

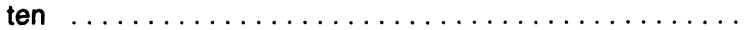

c. Wichtige Teilanalysen innerhalb des Planungs- und Analysesystems

2. Informationsbereitstellung durch das bankbetriebliche

Führungsinformationssystem ................

a. Aussagefähigkeit eines Führungsinformationssystems ...

b. Unterschiedliche Berichtstypen und Berichtsübermittlung

c. Entscheidungsfunktion des Systems ............

d. Zusammenführung der Möglichkeiten der Informationsbereitstellung

V. Hinweise zur Realisierung bankbetrieblicher Führungsinformationssysteme

A. Erfolgsfaktoren bei der Einführung bankbetrieblicher Führungsin-

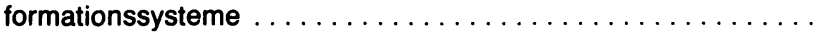

1. Notwendigkeit der Planung $\ldots \ldots \ldots \ldots \ldots \ldots \ldots \ldots \ldots$

2. Unterstützung durch das Top Management $\ldots \ldots \ldots \ldots \ldots \ldots$

3. Frühzeitiger Einbezug der Benutzer $\ldots \ldots \ldots \ldots \ldots \ldots \ldots \ldots .237$

4. Richtige Zusammensetzung des Projektteams . . . . . . 238

5. Klare Projektverantwortlichkeit und -kontrolle . . . . . . 240

6. Unterteilung des Projektes in einzelne in sich geschlossene

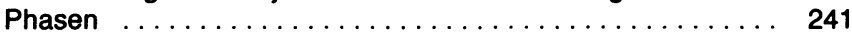

7. Situativ-kooperative Führung $\ldots \ldots \ldots \ldots \ldots \ldots \ldots \ldots, 242$

8. ${ }_{\text {Pflege }}$ des Führungsinformationssystems $\ldots \ldots \ldots \ldots \ldots 243$

B. Institutionelle Eingliederung bankbetrieblicher Führungsinforma-

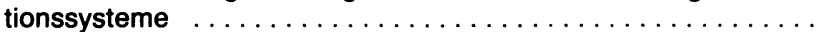

1. Eingliederung in das bankbetriebliche Controlling ..........

2. Integration, Zentralisation oder Dezentralisation der Verantwortlichkeit für das Führungsinformationssystem . . . . . . 250

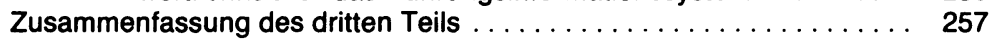

Teil Vier: Strategische Relevanz bankbetrieblicher Führungsinformationssysteme

Vorbemerkungen zum vierten Teil

I. Führungsinformationssysteme aus Sicht der bankbetrieblichen Praxis 267

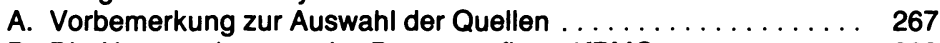

B. Die Untersuchungen der Beratungsfirma KPMG . . . . . . 268

C. Die Untersuchungen der Beratungsfirma Arthur Andersen ... . . 272

D. Die Studien der Beratungsfirma Touche Ross International ... . . 276

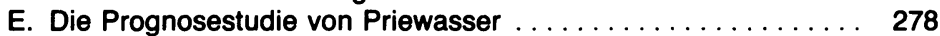

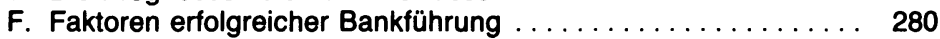

G. Charakteristika erfolgreicher Banken in der Schweiz . . . . . . 282

H. Marktuntersuchung des IHA Instituts für Marktanalysen . . . . . 283 
II. Aufgaben und Ziele der strategischen Führung in Banken . . . . . . 284

A. Wesen und Inhalt der strategischen Führung in Banken ...... 284

B. Strategische Planung als Mittelpunkt der strategischen Führung 290

III. Wettbewerbsvorteile durch Einsatz bankbetrieblicher Führungsinformationssysteme

A. Erringung von Wettbewerbsvorteilen als Ziel der strategischen Führung

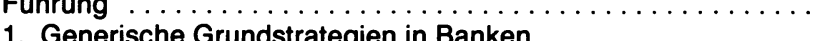

1. Generische Grundstrategien in Banken . . . . . . . . . . 294

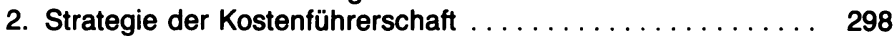

3. Strategie der Differenzierung . . . . . . . . . . . . . . . . . . 299

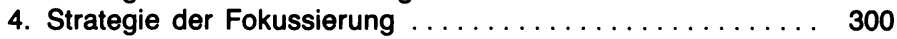

B. Strategische Einsatzmöglichkeiten bankbetrieblicher Führungsin-

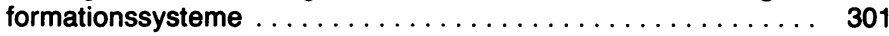

1. Notwendigkeit der Ertragsorientierung . ......... 305

2. Konsequente Markt- und Kundenorientierung ........ 309

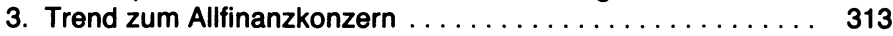

4. Anpassung bestehender Organisationsstrukturen ...... 315

5. Asset \& Liability Management ................ 320

6. Einbezug der Unternehmenskultur in die Führung . . . . . . 325

C. Führungsinformationssysteme als strategische Erfolgsposition . 328

D. Führungsinformationssysteme als strategische Waffe ...... 332

Zusammenfassung des vierten Teils $\ldots \ldots \ldots \ldots \ldots \ldots \ldots \ldots . \ldots \ldots$

Zusammenfassung und SchluBbemerkungen $\ldots \ldots \ldots \ldots \ldots 343$

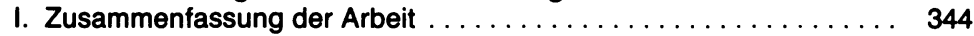

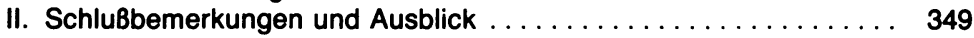

Literaturverzeichnis $\ldots \ldots \ldots \ldots \ldots \ldots \ldots \ldots \ldots \ldots \ldots \ldots \ldots \ldots \ldots$ 


\section{Abbildungsverzeichnis}

1 Beschäftigungsentwicklung im Vier-Sektoren-Modell (1882-2000) . 3

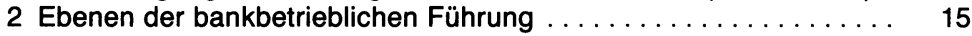

3 Aufgaben der bankbetrieblichen Führung ............. 18

4 Funktionen der bankbetrieblichen Führung $\ldots \ldots \ldots \ldots \ldots \ldots \ldots 20$

5 Institutionen der bankbetrieblichen Führung $\ldots \ldots \ldots \ldots \ldots \ldots 23$

6 Arbeits- und Aufgabeninhalte der Führungsstufen . . . . . . . . . 24

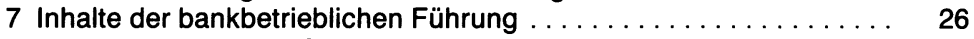

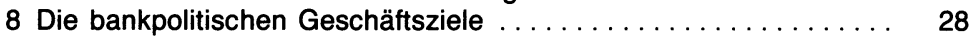

9 Einteilungsmöglichkeiten strategischer Geschäftsfelder in Banken . 36

10 Mögliche Unterteilungsmerkmale von Geschäftsziel und Teilzielen . $\quad 38$

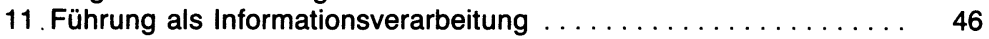

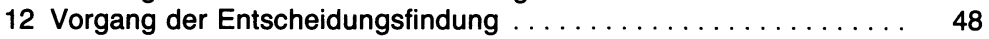

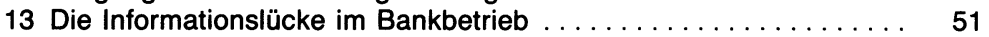

14 Das Qualitätsproblem der Information ............... 53

15 Die Elemente der bankbetrieblichen Führung im Überblick . . . . . . 56

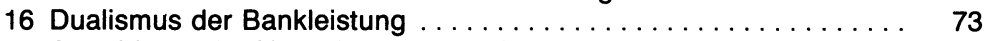

17 Auswirkung der Umweltdynamik auf die Führung . . . . . . . . 75

18 EinfluBfaktoren auf die Banken .................. 76

19 Der Anstieg der Komplexität des Bankmanagements . . . . . . . . 89

20 Auswirkungen des Wandels der Finanzmärkte auf die bankbetriebliche Informationsproblematik ......................

21 Gesamthafte Informationsintensität eines Geschäftes oder einer Branche

22 Umweltveränderung, Komplexität und Bedeutung eines bankbetrieblichen Informationsmanagements . . . . . . . . . . . . . . . . .

23 Elemente eines bankbetrieblichen Informationsmanagements .....

24 Die wichtigsten Anforderungen an ein zukunftsgerichtetes „Management der Informationen" in Banken ...................

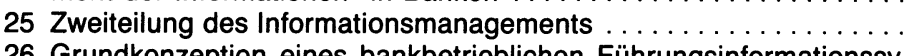

26 Grundkonzeption eines bankbetrieblichen Führungsinformationssy-

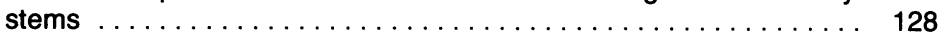

27 Aktivitäten der einzelnen Managementstufen . . . . . . . . . . . . . . . 134

28 Informationsdimensionen nach Managementebenen . . . . . . . . 137

29 Mögliche Informationskombinationen innerhalb eines bankbetriebli-

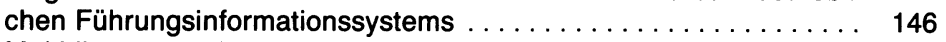

30 Multidimensionalität des bankbetrieblichen Führungsinformationssy-

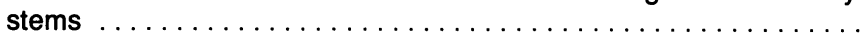

31 Manipulationsformen (M) bei der Bereitstellung von Führungsinforma-

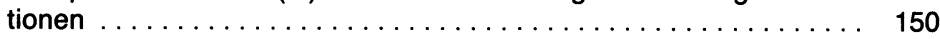

32 Risiken im Bereich der Datensicherheit . . . . . . . . . . . . . 153

33 Verfahren der Informationsbedarfsanalyse . . . . . . . . . 156

34 Diskrepanz zwischen Informations-Angebot, -Bedarf und -Nachfrage 158

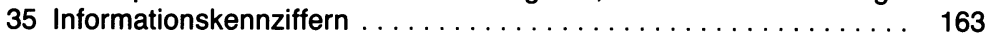

36 Beispiel eines Beziehungsdiagramms für eine schweizerische Großbank (stark vereinfacht) $\ldots \ldots \ldots \ldots \ldots \ldots \ldots \ldots \ldots \ldots \ldots$ 
37 Inhaltliche Informationsbedürfnisse des Bankmanagements (I) . . . 170

38 Inhaltliche Informationsbedürfnisse des Bankmanagements (II) . . . 171

39 Aufspaltung des Gesamtergebnisses in aussagekräftige Teilergebnisse ....

40 Aufteilung der bankbetrieblichen Einzelleistungen . . . . . . . . 185

41 Elemente der Bankkalkulation im Überblick . . . . . . . . . . . . . 187

42 Abgrenzung zwischen strategischer und operativer Planung . . . . 206

43 Informationsbezug der Planung ................. 208

44 Der Informationsprozess zwischen Bank und Umwelt (Input-

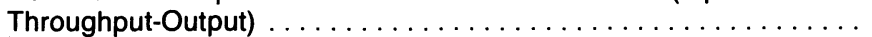

45 Ambivalenz von Planung und Analyse in der Konzeption eines bankbetrieblichen Führungsinformationssystems .............

46 Funktionen und Komplexität eines Führungsinformationssystems . .

47 Grundannahmen betreffend die Stufen eines Führungsinformationssystems

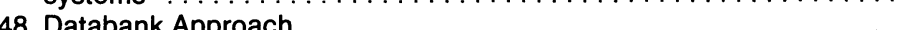

49 Predicative Information Systems $\ldots \ldots \ldots \ldots \ldots \ldots \ldots \ldots \ldots \ldots \ldots \ldots$

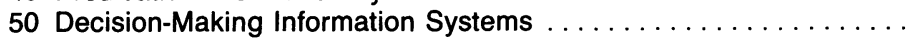

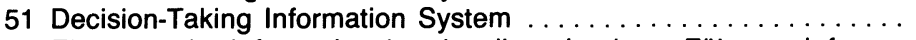

52 Elemente der Informationsbereitstellung in einem Führungsinformationssystem

53 Ziele und Zielerreichung bei der Planung von Führungsinformationssystemen

54 Drei-Phasen-Modell für die Planung und Entwicklung eines Führungsinformationssystems

55 Die Dimensionen des Bankcontrolling $\ldots \ldots \ldots \ldots \ldots \ldots \ldots \ldots \ldots$

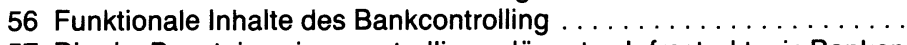

57 Die vier Bausteine einer controlling-adäquaten Infrastruktur in Banken

58 Zentrale und dezentrale Informationsrollen

59 Mögliche Informationsgrundsätze einer Bank ............

60 Durch das bankbetriebliche Führungsinformationssystem gelieferte Informationen

61 Die Konzeption des bankbetrieblichen Führungsinformationssystems

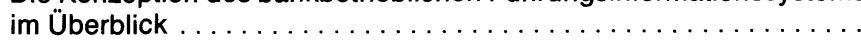

62 Antworten auf die Frage "Which needs do you think your MIS serves adequately?"

63 Antworten auf die Frage "What have been the constraining factors in developing MIS systems to meet your requirements?" .........

64 Evolution des strategischen Managements ...............

65 The Four Factor Diagram - Inhalte der strategischen Führung . . . .

66 Methoden und Verfahren in der strategischen Bankplanung .......

67 The Strategic Triangle . . . . . . . . . . . . . . . . . . .

68 Vorgehensweise bei der strategischen Planung $\ldots \ldots \ldots \ldots \ldots$

69 Drei generische Strategien . . . . . . . . . . . . . . .

70 Strategische Einsatzmöglichkeiten bankbetrieblicher Führungsinfor-

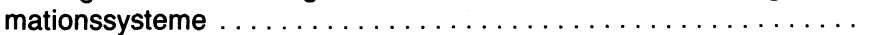

71 Strategische Verhaltensweisen der Banken ..............

72 Deckungsbeitragsorientierte Kundensegmentierung mittels eines Führungsinformationssystems $\ldots \ldots \ldots \ldots \ldots \ldots \ldots \ldots$

73 Verknüpfung von Kalkulation und Kundendaten $\ldots \ldots \ldots \ldots \ldots \ldots . \ldots \ldots$ 
74 Elemente des Risikomanagements

75 VerhaltensmäBige Möglichkeiten der Kulturorientierung und Profilierung einer Bank

76 Strategische Bedeutung des Einsatzes von Führungsinformationssystemen

77 Unterstützungsmöglichkeiten bankbetrieblicher strategischer Verhaltensweisen durch Führungsinformationssysteme

340 